\title{
Perivascular Adipose Tissue Contributes to the Modulation of Vascular Tone in vivo
}

\author{
Sophie N. Saxton ${ }^{a}$ Sarah B. Withers ${ }^{a, b}$ Jakob Nyvad ${ }^{c} \quad$ Aleksandra Mazur \\ Vladimir Matchkov ${ }^{c}$ Anthony M. Heagerty ${ }^{a}$ Christian Aalkjær ${ }^{c, d}$

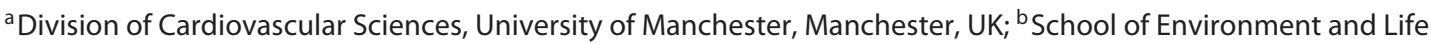 \\ Sciences, University of Salford, Salford, UK; ' ${ }^{c}$ Department of Biomedicine, Aarhus University, Aarhus, Denmark; \\ ${ }^{\mathrm{d}}$ Department of Biomedical Sciences, Copenhagen University, Copenhagen, Denmark
}

\section{Keywords}

Adipocytes · Adrenoceptors · Vascular tone

\begin{abstract}
Background: Perivascular adipose tissue (PVAT) reduces vascular tone in isolated arteries in vitro, however there are no studies of PVAT effects on vascular tone in vivo. In vitro adipocyte $\beta_{3}$-adrenoceptors play a role in PVAT function via secretion of the vasodilator adiponectin. Objective: We have investigated the effects of PVAT on vessel diameter in vivo, and the contributions of $\beta_{3}$-adrenoceptors and adiponectin. Method: In anaesthetised rats, sections of the intact mesenteric bed were visualised and the diameter of arteries was recorded. Arteries were stimulated with electrical field stimulation (EFS), noradrenaline (NA), arginine-vasopressin (AVP), and acetylcholine (Ach). Results: We report that in vivo, stimulation of PVAT with EFS, NA, and AVP evokes a local anti-constrictive effect on the artery, whilst PVAT exerts a pro-contractile effect on arteries subjected to Ach. The anticonstrictive effect of PVAT stimulated with EFS and NA was significantly reduced using $\beta_{3}$-adrenoceptor inhibition, and activation of $\beta_{3}$-adrenoceptors potentiated the anti-con-
\end{abstract}

\section{KARGER}

(C) 2019 S. Karger AG, Basel

E-Mail karger@karger.com

www.karger.com/jvr strictive effect of vessels stimulated with EFS, NA, and AVP. The $\beta_{3}$-adrenoceptor agonist had no effect on mesenteric arteries with PVAT removed. A blocking peptide for adiponectin receptor 1 polyclonal antibody reduced the PVAT anti-constrictive effect in arteries stimulated with EFS and NA, indicating that adiponectin may be the anti-constrictive factor released upon $\beta_{3}$-adrenoceptor activation. Conclusions: These results clearly demonstrate that PVAT plays a paracrine role in regulating local vascular tone in vivo, and therefore may contribute to the modulation of blood pressure. This effect is mediated via adipocyte $\beta_{3}$-adrenoceptors, which may trigger release of the vasodilator adiponectin.

(c) 2019 S. Karger AG, Basel

\section{Introduction}

Perivascular adipose tissue (PVAT) surrounds the majority of the peripheral vasculature and there is substantial evidence that this tissue releases adipokines which contribute to modulation of vascular tone in vitro [1-6]. There have been no studies to investigate the direct effects of PVAT on vascular tone in vivo; however, in coronary 
artery bypass surgeries saphenous vein grafts with PVAT intact exhibit a superior patency rate, and better preserved intimal, medial, and adventitial architecture compared to veins with PVAT removed $[7,8]$. This suggests that PVAT does have beneficial effects in vivo. The adipokines released from PVAT are present in the circulation; therefore, there is debate as to whether PVAT exerts a local paracrine effect in vivo on the vasculature it surrounds, as opposed to an endocrine effect of the circulating adipokines. In obesity, PVAT function is lost, and whilst there is no direct evidence that loss of PVAT function contributes to hypertension, there is a correlation between PVAT dysfunction and elevated blood pressure [9-11]. Therefore, there is a need to definitively determine the local influence of PVAT on vascular tone in vivo to further study the mechanistic links between PVAT dysfunction in obesity and hypertension.

Recently, we have demonstrated that sympathetic stimulation of PVAT in vitro elicits an anti-contractile effect on the vasculature [12]. This effect is mediated via adipocyte $\beta_{3}$-adrenoceptors and the NE (norepinephrine) transporter organic cation transporter 3 (OCT3). Via OCT3, PVAT sequesters some nerve-derived NE, thereby preventing the NE from stimulating contraction of the vascular smooth muscle [12]. Activation of $\beta_{3^{-}}$ adrenoceptors triggers the release of the vasodilator adiponectin. Adiponectin is almost exclusively secreted by adipocytes, and in both obesity and hypertension bioavailability of adiponectin is reduced $[13,14]$, indicating a possible role for adipocyte-derived adiponectin in blood pressure regulation. In obesity, adipose tissue becomes inflamed, which is likely the cause for adipokine dysregulation $[4,15,16]$. Inflammation of adipose tissue is common to both lean and obese hypertensive mice, which is convincing evidence that the inflammatory state of adipose tissue is implicated in vascular dysfunction in obesity [17]. Again, this highlights a need to study PVAT function in vivo.

We have validated a novel approach to the intravital study of rat mesenteric arterial tone [18]. In anaesthetised rats, we are able to exteriorise a section of the small intestine, and isolate an intact segment of a small mesenteric artery in a small chamber. The artery diameter can then be recorded using a camera mounted on to a microscope. This preparation is crucial to understand the physiological importance of adipocyte-derived factors in modulating vascular tone.

In the present study, we aim to determine the contribution of PVAT to vascular tone in vivo. We will investigate whether the mechanisms we have recently described behind the PVAT anti-contractile effect in vitro are physiologically relevant, which will reinforce the importance of studies on the contribution of PVAT to vascular diseases in obesity.

\section{Materials and Methods}

\section{Animal Care and Handling}

All experiments were approved by and conducted with permission from the Animal Experiments Inspectorate of the Danish Ministry of Justice. Male Wistar rats (Janvier Labs, France) were maintained on a 12-h light/dark schedule, and were given water and standard chow ad libitum. Rats were aged 12-15 weeks and weighed $400-550 \mathrm{~g}$.

Unfasted rats were anaesthetized with a subcutaneous injection of ketamine $(33 \mathrm{mg} / 100 \mathrm{~g}$; Ketaminol vet, Intervet International, The Netherlands) and xylazine $(7.5 \mathrm{mg} / 100 \mathrm{~g}$; Rompun vet, Bayer, Germany). To maintain anaesthesia, rats were given supplementary intramuscular injections. It has been proven that the whisker and the neck region of the rat motor cortex are highly sensitive to the depth of anaesthesia [19], therefore movement of the whiskers was determined to be a prompt to inject supplementary doses of anaesthetic.

\section{Visualizing and Recording Arterial Responses in vivo}

Anaesthetised rats were placed on a heating plate at $37^{\circ} \mathrm{C}$, and a $1-\mathrm{cm}$ incision was made in the left lower quadrant of the abdomen. A short segment $(3-5 \mathrm{~cm})$ of the small intestine and the mesentery were carefully exposed. Connective tissue surrounding a single branch of the mesenteric artery $(<200 \mu \mathrm{m})$ and the attached vein were dissected away, and the branch was slotted into a small reservoir $(6.5 \mathrm{~mm}$ width $\times 6.5 \mathrm{~mm}$ length $\times 3 \mathrm{~mm}$ depth, corresponding to approx. $200 \mu \mathrm{L}$ ) by passing ends of the branch through openings in the two sides of the reservoir. The openings between the reservoir walls and the branch were sealed with high vacuum grease (Dow Corning GmbH, Wiesbaden, Germany; Fig. 1a, b). The mesenteric artery segment in the reservoir was thereby isolated from the rest of the intestine and the mesentery. The reservoir was filled with physiological salt solution (PSS; containing in mM: $119 \mathrm{NaCl}, 2.82 \mathrm{KCl}, 1.18 \mathrm{KH}_{2} \mathrm{PO}_{4}, 1.17 \mathrm{MgSO}_{4}, 25 \mathrm{NaHCO}_{3}, 1.6$ $\mathrm{CaCl}_{2}, 0.026 \mathrm{EDTA}, 5.5$ glucose, and 10 Hepes) kept at $37^{\circ} \mathrm{C}$ and gassed with $5 \% \mathrm{CO}_{2} /$ air to adjust to $\mathrm{pH}$ 7.4. This preparation is described in more detail by Nyvad et al. [18].

For PVAT-denuded experiments, $90 \%$ of surrounding adipose tissue was dissected away from the artery and vein, although a small amount remained at the two openings of the reservoir. For PVAT-intact experiments, a small amount of the overlaying adipose tissue on the artery was removed; just enough to visualize the artery walls. During dissection and the experiment, the extracted segment of the surrounding intestine and the mesentery was kept moist using regular washings with PSS, and was covered using parafilm to prevent evaporation and heat loss (Fig. 1a, b).

The artery in the reservoir was observed through a microscope (Motic PSM-1000, China). The arterial inner diameter was visualized with a USB CCD Monochrome Camera (DMK 41AU02, Imaging Source, Germany) attached to the microscope. Artery diameter was recorded and processed using DMT Vessel Acquisition Suite software (Danish Myo Technology A/S, Denmark). 


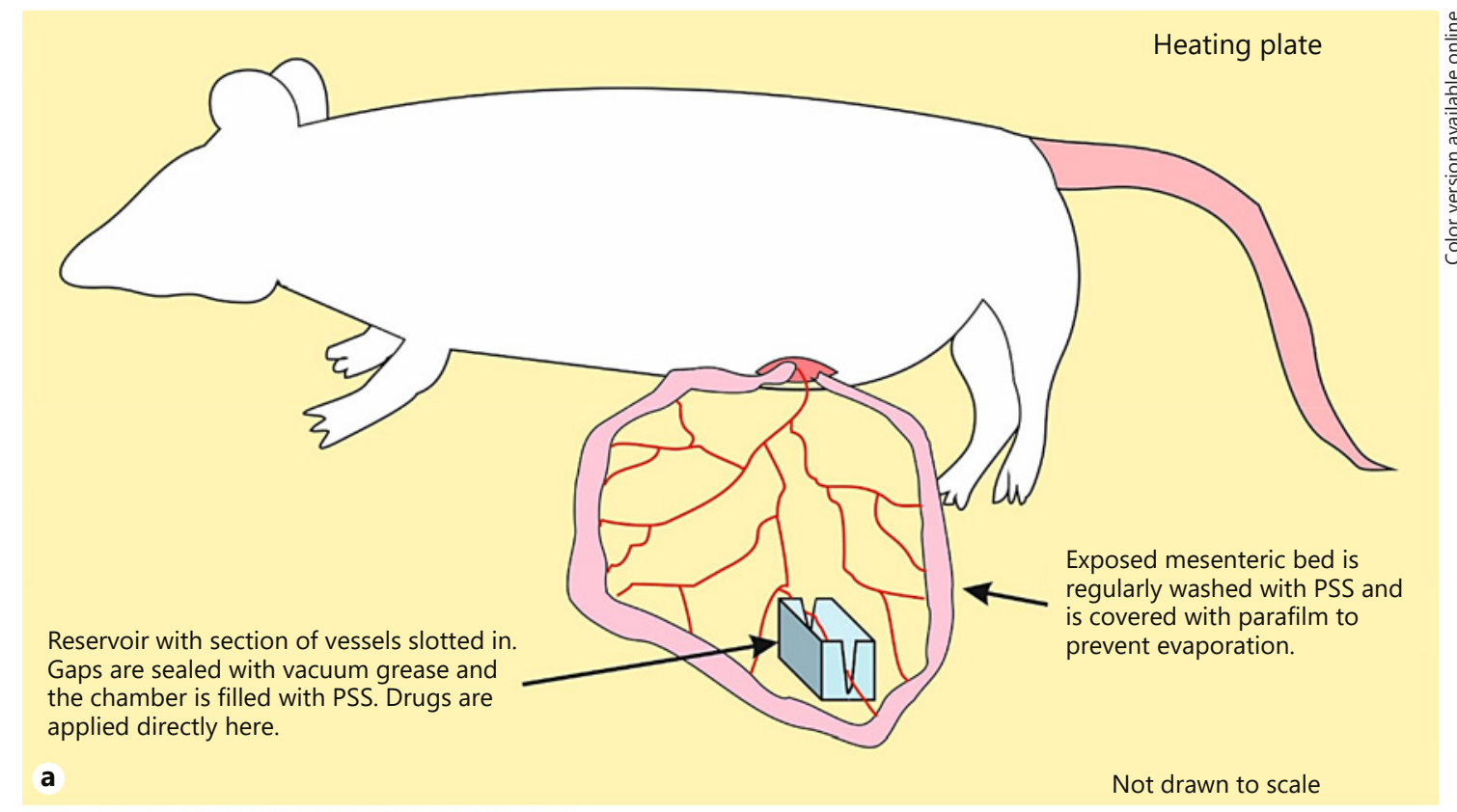

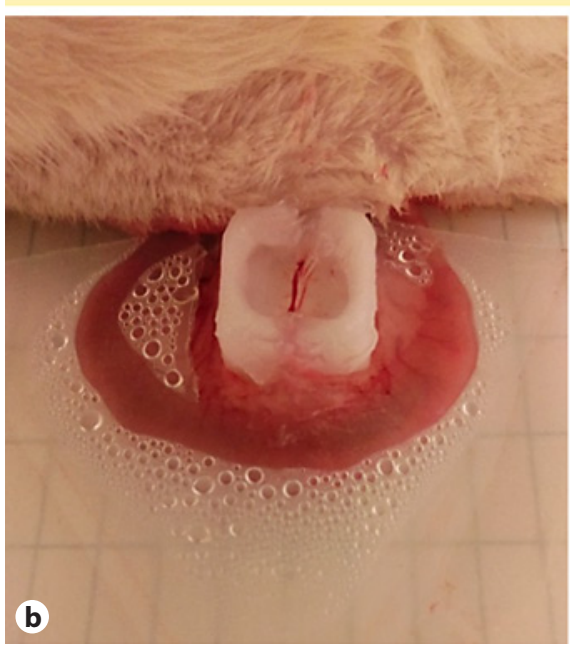

Electrical Field Stimulation and Concentration-Response Curves

For electrical field stimulation (EFS), two platinum electrodes were lowered into the reservoir on either side of the vessels. The arteries were stimulated at a frequency of $2-32 \mathrm{~Hz}$ (pulse width 1.5 $\mathrm{ms}$, current $15 \mathrm{~mA}$, train duration $10 \mathrm{~s}$ ), with $20 \mathrm{~s}$ recovery between each frequency. The contractile responses up to $16 \mathrm{~Hz}$ were fully blocked by tetrodotoxin, and after treatment with guanethidine [20].

Cumulative concentration-response curves were generated to $\mathrm{NE}\left(1 \times 10^{-7}\right.$ to $\left.3 \times 10^{-5} \mathrm{M}\right)$, acetylcholine (Ach; $1 \times 10^{-8}$ to $1 \times$ $\left.10^{-5} \mathrm{M}\right)$, and arginine-vasopressin (AVP; $1 \times 10^{-5}$ to $3 \times 10^{-2} \mathrm{M}$ ). Drugs were applied locally and given in half molar increments, and were administered when constriction reached a plateau (2- to 10min intervals). For the Ach concentration response, arteries were first preconstricted to $60 \%$ of their internal diameter using NE. The same order of stimuli was repeated in each experiment, i.e., EFS, noradrenaline (NA), Ach, and then AVP.
Fig. 1. Vessel preparation. a A 1-cm incision is made in the abdomen of the anaesthetised rat and the mesentery is exposed. The exposed small intestine is covered with parafilm to prevent drying out. A branch of the mesentery is slotted into the reservoir, and the ends are sealed with vacuum grease. The reservoir is filled with PSS, and drugs are applied directly here. $\mathbf{b}$ In this preparation, the PVAT has been removed, and the end of a small pipette tip has been used to aid separation of the vessels, without disturbing blood flow. The vein is on the left, and the artery is on the right.

\section{Pharmacological Tools}

A number of pharmacological tools were used to elucidate the PVAT mechanism. Control measurements in PVAT-denuded and intact arteries were recorded to EFS, NE, Ach, and AVP, before incubation with the appropriate drug. Drugs were applied locally, directly into the vessel chamber (Fig. 1a). To investigate the role of $\beta_{3}$-adrenoceptors, the agonist CL-316,243 (10 $\mu \mathrm{M}$, cat. No. 1499; Bio-Techne, Abingdon, UK) and antagonist SR59230A ( $1 \mu \mathrm{M}$, cat. No. 1151; Bio-Techne) were used. These were dissolved in PSS and arteries were incubated with these compounds for $30 \mathrm{~min}$. To examine the contribution of the vasodilator adiponectin, a blocking peptide for adiponectin receptor 1 polyclonal antibody (ABP; $3.5 \mu \mathrm{g} / \mathrm{mL}$, cat. No. ALX-151-045-C100; Enzo Life Sciences, Exeter, UK) was used. As a control for ABP, a blocking peptide with a similar size and charge was used: blocking peptide for toll-like receptor 4 polyclonal antibody (TRBP; $3.5 \mu \mathrm{g} / \mathrm{mL}$, cat. No. ALX-168-014; Enzo Life Sciences). Following incubation, all control measurements were repeated. Only one 


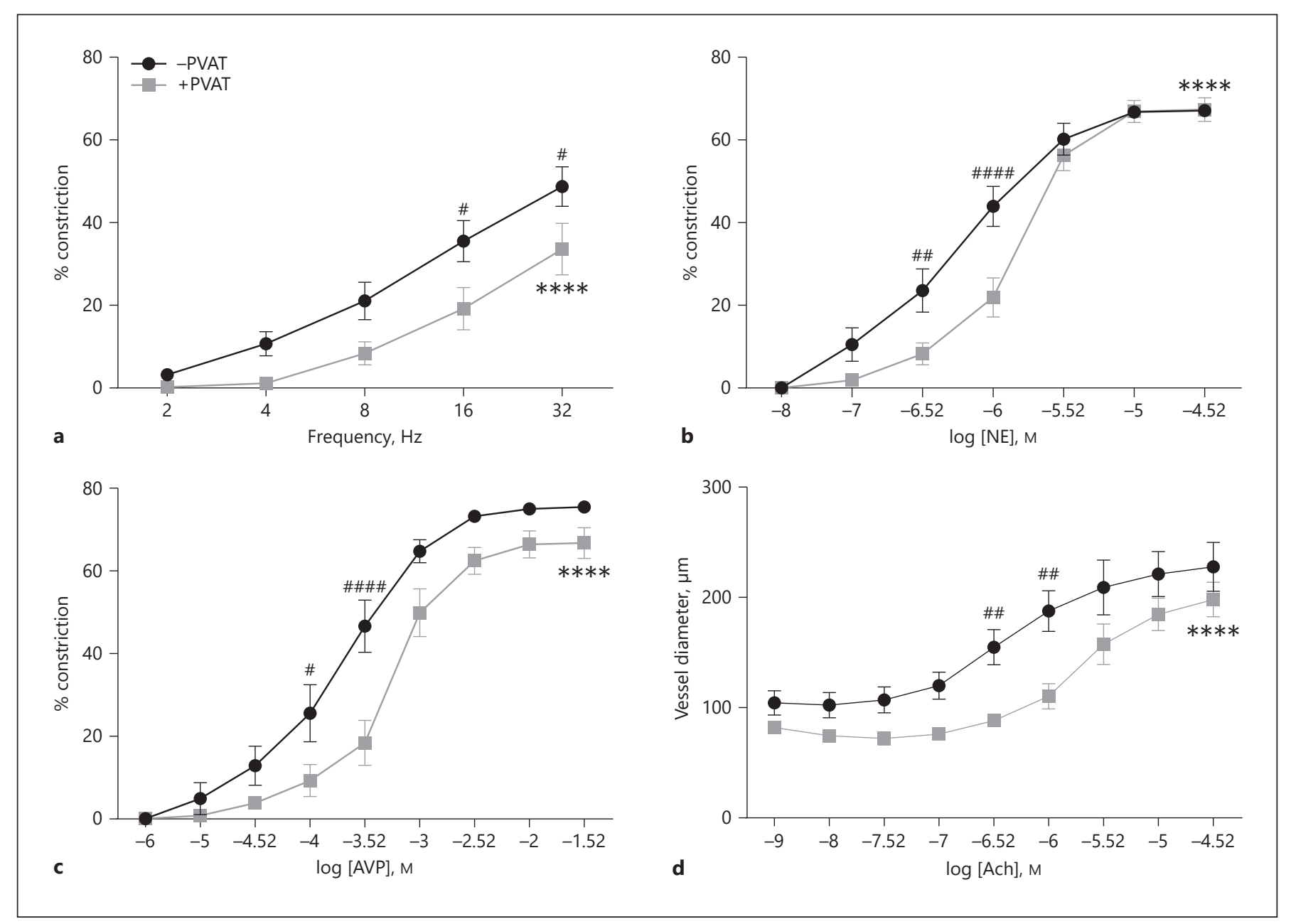

Fig. 2. The presence of PVAT alters artery constriction in vivo. Rat mesenteric resistance arteries with and without PVAT were challenged with EFS $(2-32 \mathrm{~Hz} ; \mathbf{a})$, NE $\left(1 \times 10^{-7}\right.$ to $\left.3 \times 10^{-5} \mathrm{M} ; \mathbf{b}\right)$, and $\operatorname{AVP}\left(1 \times 10^{-5}\right.$ to $\left.3 \times 10^{-2} \mathrm{M} ; \mathbf{c}\right)$. For the Ach concentration response $\left(1 \times 10^{-8}\right.$ to $1 \times 10^{-5} \mathrm{M}$; d), vessels were first preconstricted with NE. Data shown are the mean \pm SEM. Differences in curves were tested using two-way ANOVA $\left(n=10,{ }^{* * * *} p<0.0001\right)$ with Bonferroni post hoc tests ( ${ }^{\#} p<0.05$; ${ }^{\# \#} p<0.01$; $\left.{ }^{\# \# \# ~} p<0.0001\right)$. agonist/antagonist was used in each experiment, i.e., new vessels were used for each intervention.

In vivo Rat Mesenteric Resistance Artery Vessel Measurements

Arterial responses to EFS, NE, and AVP were expressed as a percentage change from the inner diameter baseline, recorded at rest before each protocol. The vasodilation elicited with Ach is presented as the raw vessel diameter measurements. Differences between the constrictions of PVAT arteries with and without PVAT were tested using a two-way ANOVA, with a Bonferroni post hoc test. Before and after treatments within the same vessel were compared using a repeated-measures ANOVA, again followed by a Bonferroni post hoc test. Normal distribution was confirmed using the Shapiro-Wilk normality test, supported by skewness and kurtosis coefficients (using an acceptable range of $\pm 2)$.

PVAT Effect in vivo

\section{Results}

PVAT Exerts Local Effects on Vessel Diameter in vivo

To determine if the PVAT anti-contractile effect is present in vivo, rat mesenteric arteries with and without PVAT were challenged with EFS $(2-32 \mathrm{~Hz}), \mathrm{NE}\left(1 \times 10^{-7}\right.$ to $\left.3 \times 10^{-5} \mathrm{M}\right)$, and AVP $\left(1 \times 10^{-5}\right.$ to $\left.3 \times 10^{-2} \mathrm{M}\right)$. For the Ach concentration response $\left(1 \times 10^{-8}\right.$ to $\left.1 \times 10^{-5} \mathrm{M}\right)$, arteries were preconstricted with NE to $60 \%$ of their maximal internal diameter (determined using the previous NA concentration response in the same artery). The presence of PVAT significantly reduced constriction elicited with EFS, NE, and AVP $(p<0.0001, n=10$; Fig. 2a-c). The dilation to Ach was also significantly reduced $(p<$ 


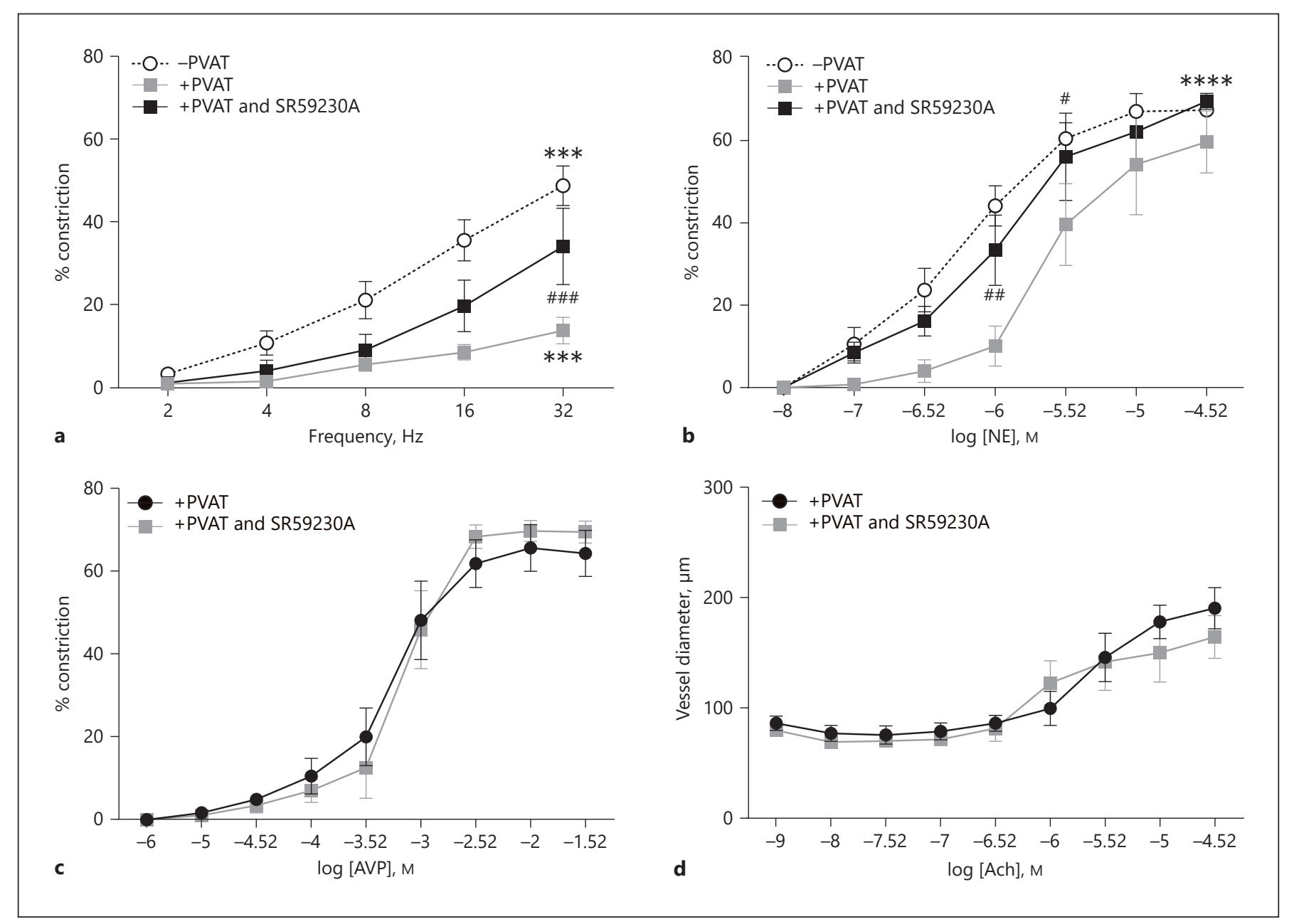

Fig. 3. $\beta_{3}$-Adrenoceptor inhibition reduces PVAT function in arteries stimulated with EFS and NE in vivo. Following control stimulations to EFS (a), NE (b), AVP (c), and Ach (d), arteries with PVAT intact were incubated with $\beta_{3}$-adrenoceptor antagonist SR59230A $(1 \mu \mathrm{M})$ for $30 \mathrm{~min}$. The control responses of PVAT- denuded arteries to EFS and NE shown in Figure 2 were superimposed on to graphs $\mathbf{a}$ and $\mathbf{b}$. Data shown are the mean \pm SEM. Differences in curves were tested using repeated-measures ANOVA $\left(n=6,{ }^{* * *} p<0.001,{ }^{* * * *} p<0.0001\right)$ with Bonferroni post hoc tests $\left({ }^{\#} p<0.05\right.$; \#\# $p<0.01$; \#\# $\left.p<0.001\right)$.
$0.0001, n=10$; Fig. 2 d). Post hoc tests revealed that PVAT significantly reduced the maximal response with EFS $(p<0.05)$, whereas there was no difference in the maximal constriction between arteries with and without PVAT in response to NE, AVP, and Ach. These results suggest that PVAT does modulate artery diameter in vivo.

In these experiments, protocols were first conducted in arteries with PVAT intact. The fat was then removed from the section of artery within the bath, but was left intact upstream and downstream of the bath. Therefore, the increase in contractility when the fat was removed from the bath suggests that PVAT exerts a paracrine effect.
Adipocyte $\beta_{3}$-Adrenoceptors Contribute to the PVAT Anti-Contractile Effect of EFS- and NE-Stimulated Arteries

Previously, we have demonstrated in mouse mesenteric arteries that the in vitro PVAT anti-contractile effect is dependent upon $\beta_{3}$-adrenoceptors [12, 21]. Therefore, we examined the role of $\beta_{3}$-adrenoceptors in vivo in rat mesenteric small arteries.

\section{$\beta_{3}$-Adrenoceptor Antagonist}

Following control stimulations with EFS, NE, AVP, and Ach, rat mesenteric arteries were incubated with $1 \mu \mathrm{M}$ of SR59230A for $30 \mathrm{~min}$. In arteries with PVAT intact, 


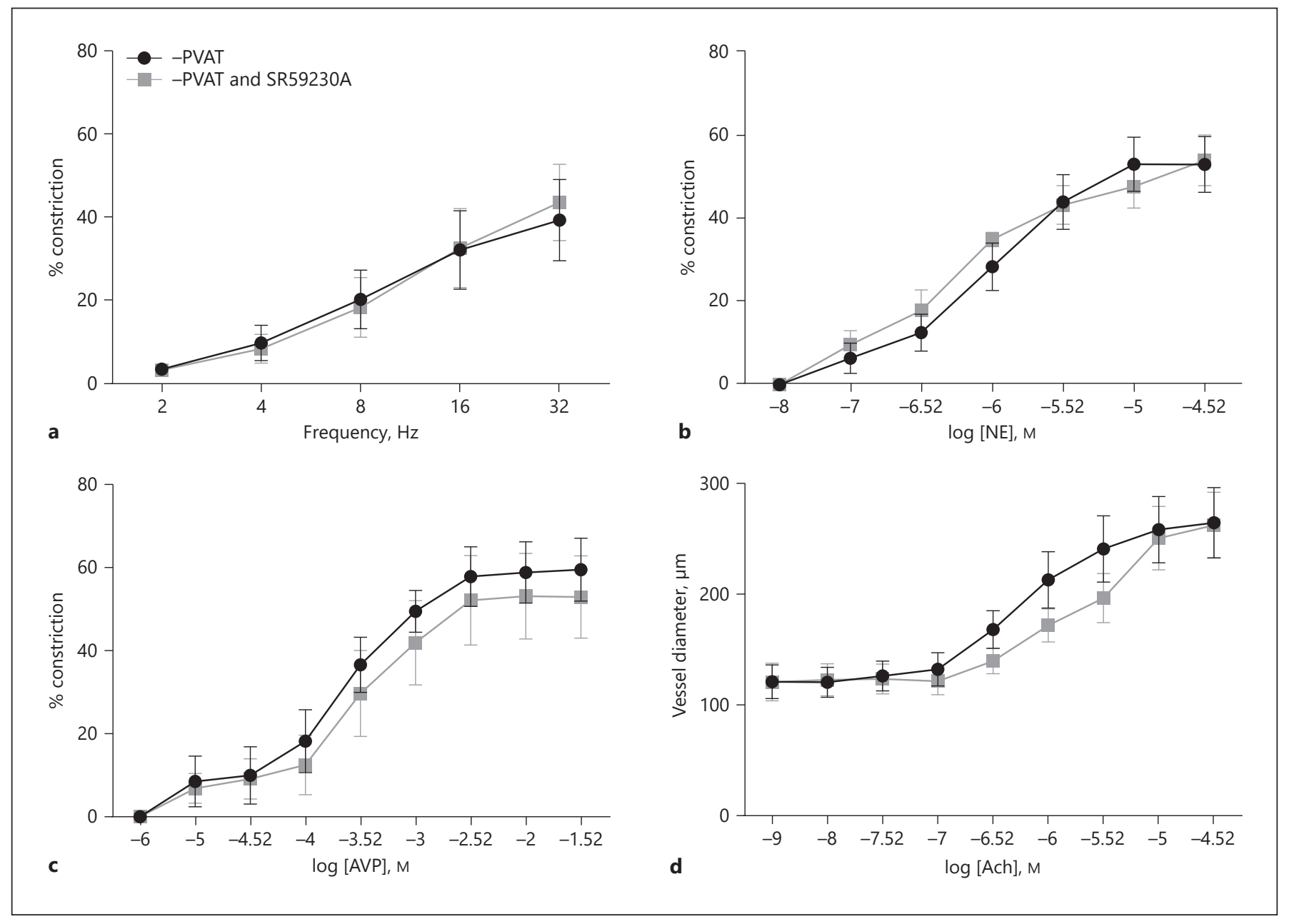

Fig. 4. $\beta_{3}$-Adrenoceptor inhibition had no effect on PVAT-denuded arteries in vivo. Following control stimulations with EFS (a), NE (b), AVP (c), and Ach (d), arteries with PVAT removed were incubated with SR59230A $(1 \mu \mathrm{M})$ for $30 \mathrm{~min}$. Data shown are the mean \pm SEM. Differences in curves were tested using repeated-measures ANOVA $(n=6, p>0.05)$ with Bonferroni post hoc tests.

SR59230A significantly increased constriction to EFS $(p<0.001, n=6)$ and NE ( $p<0.0001, n=6$; Fig. 3a, b). The control constriction of PVAT-denuded arteries to EFS and NE shown in Figure 2 were superimposed on to the graphs, to determine if SR59230A inhibition returned the responses of PVAT-intact arteries to a level comparable with PVAT-denuded artery responses. SR59230A reduced the anti-constrictive effect of PVAT induced with EFS, however the constriction to EFS in PVAT-intact vessels was still significantly lower than constriction to EFS arteries without PVAT $(p<0.001$, PVAT denuded, $n=10$ ), which is consistent with previous in vitro studies [12]. For the NE concentration-response, SR59230A completely abolished the PVAT anti-constrictive effect, and the responses of PVAT-intact arteries increased to a level similar to that of PVAT-denuded arteries $(p>0.05$, PVAT denuded, $n=10$ ). SR59230A had no significant effect on the constriction of PVAT-intact arteries to AVP and Ach ( $p>0.05, n=6$; Fig. 3c, d). Similarly, SR59230A had no effect on PVAT-denuded artery responses to EFS, NE, AVP, or Ach ( $p>0.05, n=6$; Fig. 4$)$, confirming that the effects of the antagonist are specific to adipocyte $\beta_{3^{-}}$ adrenoceptors. These results indicate that the mechanism of the PVAT effect in vivo is agonist dependent, and $\beta_{3^{-}}$ adrenoceptors are involved in the sympathetic/noradrenergic PVAT anti-contractile effect.

\section{$\beta_{3}$-Adrenoceptor Agonist}

Following control stimulations with EFS, NE, AVP, and Ach, rat mesenteric arteries were incubated with CL- 


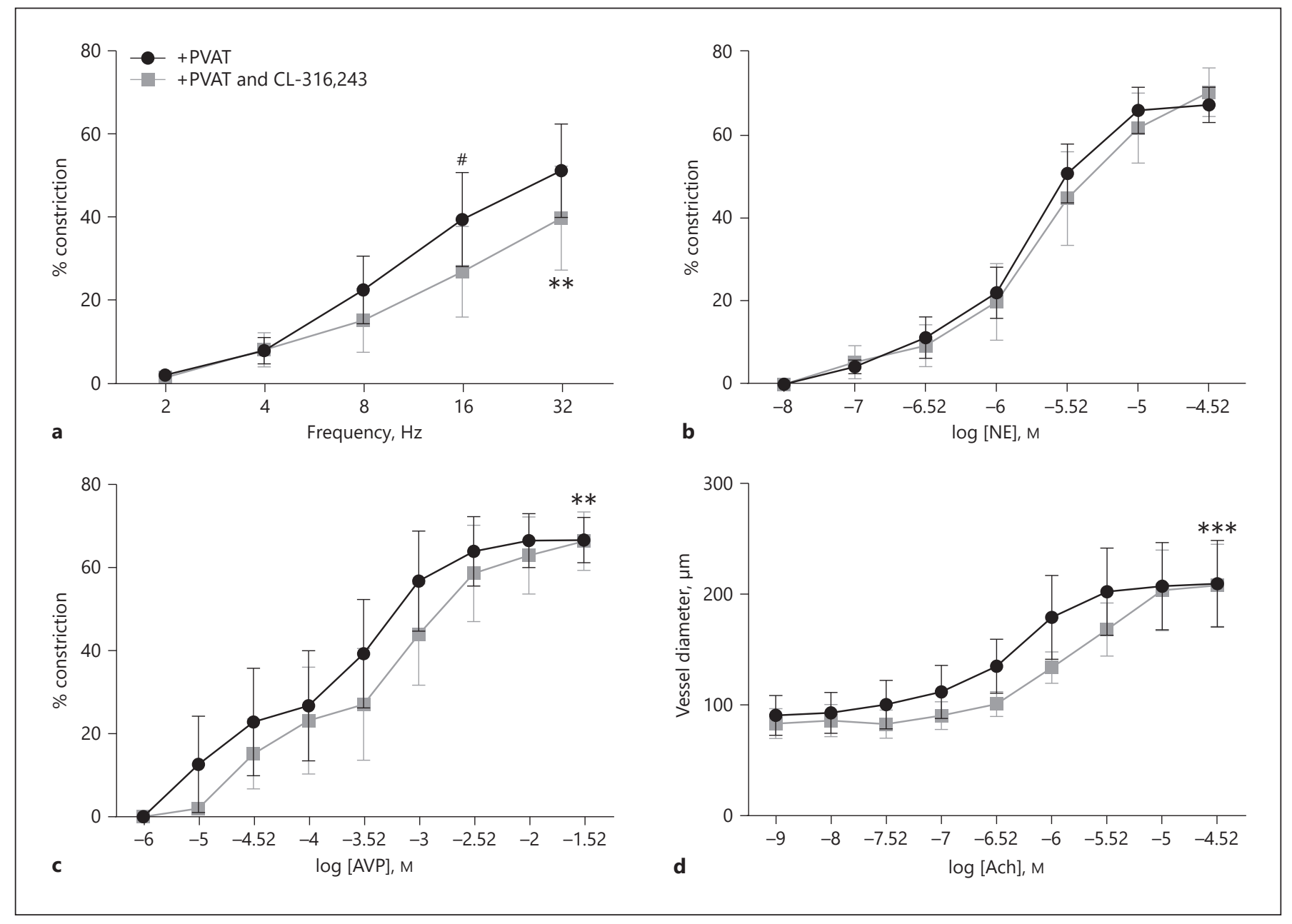

Fig. 5. $\beta_{3}$-Adrenoceptor activation potentiated the PVAT function in vivo. Following control stimulations with EFS (a), NE (b), AVP (c), and Ach (d), arteries with PVAT intact were incubated with CL-316,243 (10 $\mu \mathrm{M})$ for $30 \mathrm{~min}$. Data shown are the mean \pm SEM. Differences in curves were tested using repeated-measures ANOVA $\left(n=6,{ }^{* *} p<0.01,{ }^{* * *} p<0.001\right)$ with Bonferroni post hoc tests $\left({ }^{\#} p<0.05\right)$.

316,243 (10 $\mu \mathrm{M})$ for $30 \mathrm{~min}$. In PVAT-intact arteries, CL316,243 significantly reduced responses with EFS $(p<$ $0.01, n=6), \operatorname{AVP}(p<0.01, n=6)$, and Ach $(p<0.001$, $n=6$; Fig. $5 \mathrm{a}, \mathrm{c}, \mathrm{d})$, although Bonferroni post hoc tests indicated no differences at individual points on the concentration-response curves for AVP and Ach. At the higher concentrations of AVP and Ach, the responses of arteries incubated with CL-316,243 reached the maximal change in diameter, whereas the maximal constriction to EFS was still reduced $(p<0.05)$. CL-316,243 had no effect on the constriction to NE ( $p>0.05, n=6$; Fig. 5b). Again, these results are consistent with in vitro studies $[12,21]$, and suggest that activation of $\beta_{3}$-adrenoceptors triggers the release of an anti-contractile factor from PVAT; however, when using NE the receptors may already be satu- rated. The $\beta_{3}$-adrenoceptor agonist had no significant effect on PVAT-denuded arteries stimulated with EFS, NE, AVP, or Ach, again confirming that the effects of CL316,243 are specific to activation of adipocyte $\beta_{3}$-adrenoceptors ( $p>0.05, n=6$; Fig. 6).

\section{Inhibition of Adiponectin Signalling Reduced the EFS PVAT Anti-Constrictive Effect}

Recently, we have demonstrated that the anti-contractile factor release upon $\beta_{3}$-adrenoceptor activation is adiponectin, as the in vitro EFS anti-contractile effect was reduced by $\mathrm{ABP}$, and was absent in the adiponectin knockout mouse [12]. In addition, adiponectin secretion from PVAT is reduced in the presence of the $\beta_{3^{-}}$ adrenoceptor antagonist. Therefore, we tested the effects 


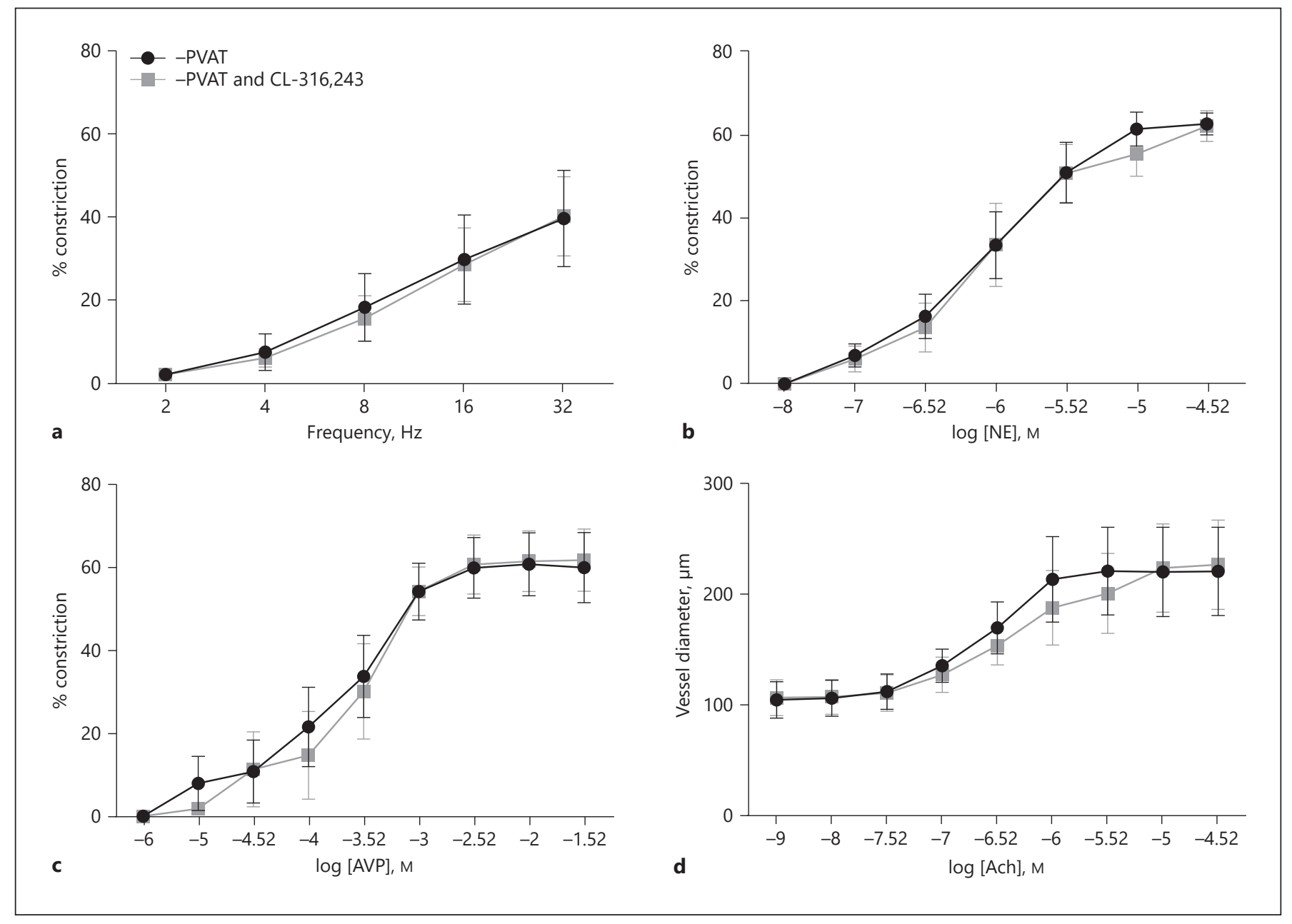

Fig. 6. $\beta_{3}$-Adrenoceptor activation had no effect on PVAT-denuded arteries in vivo. Following control stimulations with EFS (a), NE (b), AVP (c), and Ach (d), vessels with PVAT removed were incubated with CL-316,243 $(10 \mu \mathrm{M})$ for $30 \mathrm{~min}$. Data shown are the mean \pm SEM. Differences in curves were tested using repeated-measures ANOVA $(n=6, p>0.05)$ with Bonferroni post hoc tests.

of the $\mathrm{ABP}$ in vivo. Following control stimulations with EFS and NE, PVAT-intact and PVAT-denuded arteries were incubated with $\mathrm{ABP}(5 \mu \mathrm{g} / \mathrm{mL})$ for $45 \mathrm{~min}$. ABP significantly reduced the PVAT anti-constrictive effect of the EFS-stimulated arteries $(p<0.001, n=6)$, and NEstimulated arteries $(p<0.01, n=5)$, indicating that adiponectin may be the anti-constrictive factor released upon $\beta_{3}$-adrenoceptor activation (Fig. 7). ABP had no effect on PVAT-denuded arteries ( $p>0.05, n=4)$.

As a control for the ABP, the effects of a blocking peptide for an unrelated protein were tested. TRBP has a similar charge, size, and sequence to the ABP. The protocol for $\mathrm{ABP}$ was repeated exactly using TRBP; following control stimulations PVAT-intact arteries were incubated for
45 min with TRBP $(3.5 \mu \mathrm{g} / \mathrm{mL})$. Incubation with TRBP had no effect on arteries stimulated with EFS or NE ( $p>$ $0.05, n=3$; Fig. 8).

\section{Discussion}

This is the first study to define a paracrine effect of PVAT on vascular tone in vivo. The aim of this study was to investigate the role of PVAT in modulating vascular tone in vivo. The main findings of this study were: (1) stimulation of PVAT induces significant pro- and anticonstrictive paracrine effects in vivo in rat mesenteric arteries, (2) the sympathetic nerve-mediated anti-constric- 


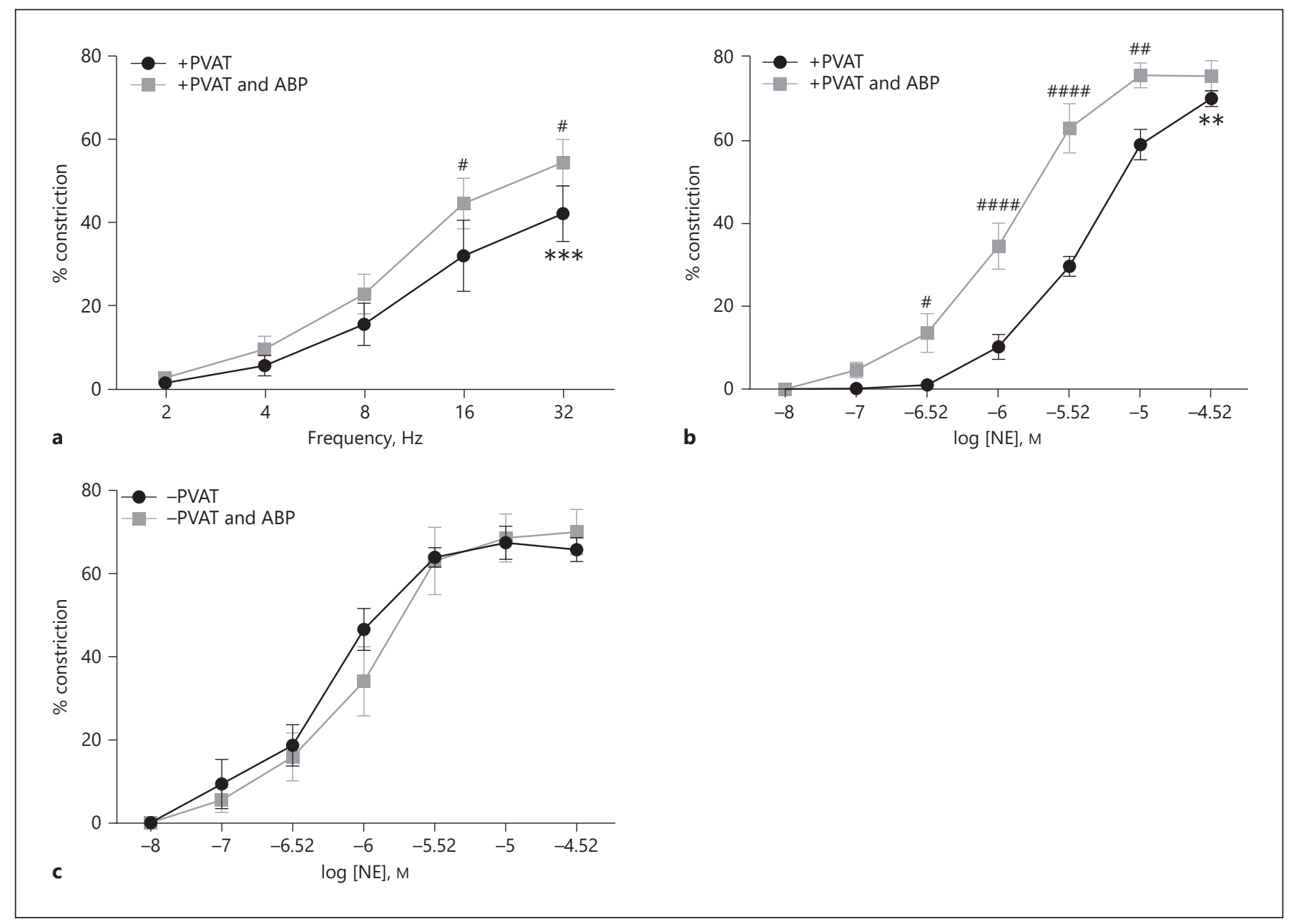

Fig. 7. ABP significantly reduced the PVAT anti-constrictive effect in vivo. Following control stimulations with EFS (PVAT intact, $n=6$; a) and NE (PVAT intact, $n=5 ; \mathbf{b}$; PVAT denuded, $n=4 ; \mathbf{c}$ ), rat mesenteric arteries were incubated with ABP $(5 \mu \mathrm{g} / \mathrm{mL})$ for $45 \mathrm{~min}$. Data shown are the mean \pm SEM. Differences in curves were tested using repeated-measures ANOVA $\left({ }^{* *} p<0.01,{ }^{* * *} p<0.001\right)$ with Bonferroni post hoc tests $\left({ }^{\#} p<0.05\right.$; ${ }^{\# \#} p<$ $\left.0.01 ;{ }^{\# \# \# ~} p<0.0001\right)$.

tive effect of PVAT is dependent on $\beta_{3}$-adrenoceptor activation in vivo, (3) adiponectin may be the anti-constrictive factor released upon $\beta_{3}$-adrenoceptor activation in vivo, and (4) $\beta_{3}$-adrenoceptors have no role in vascular tone in small mesenteric arteries devoid of PVAT.

\section{PVAT May Be Important in Maintaining Vascular}

\section{Tone in vivo}

Over the past 25 years there has been emerging evidence for the role of PVAT in modulating vascular tone in vitro $[4,22-24]$. This study has shown for the first time that the anti-contractile function of PVAT in response to vasoconstrictors is still present in vivo and mediates a lo- cal effect. Furthermore, this is the first study to show an AVP-induced anti-constrictive effect, and a proconstrictive effect of PVAT with Ach. Consistent with previously published in vitro studies, NE and EFS induced a PVAT anti-constrictive effect in vivo $[4,12,21-24]$. These effects are likely to modulate vascular resistance, and maintain blood pressure.

It has been suggested that PVAT may form a barrier around the blood vessels, and by using an exogenous PVAT preparation we have previously been able to show that this is not the case [12]. In brief, an artery without PVAT is mounted in a myograph and a section of exogenous PVAT is suspended above. When this preparation 


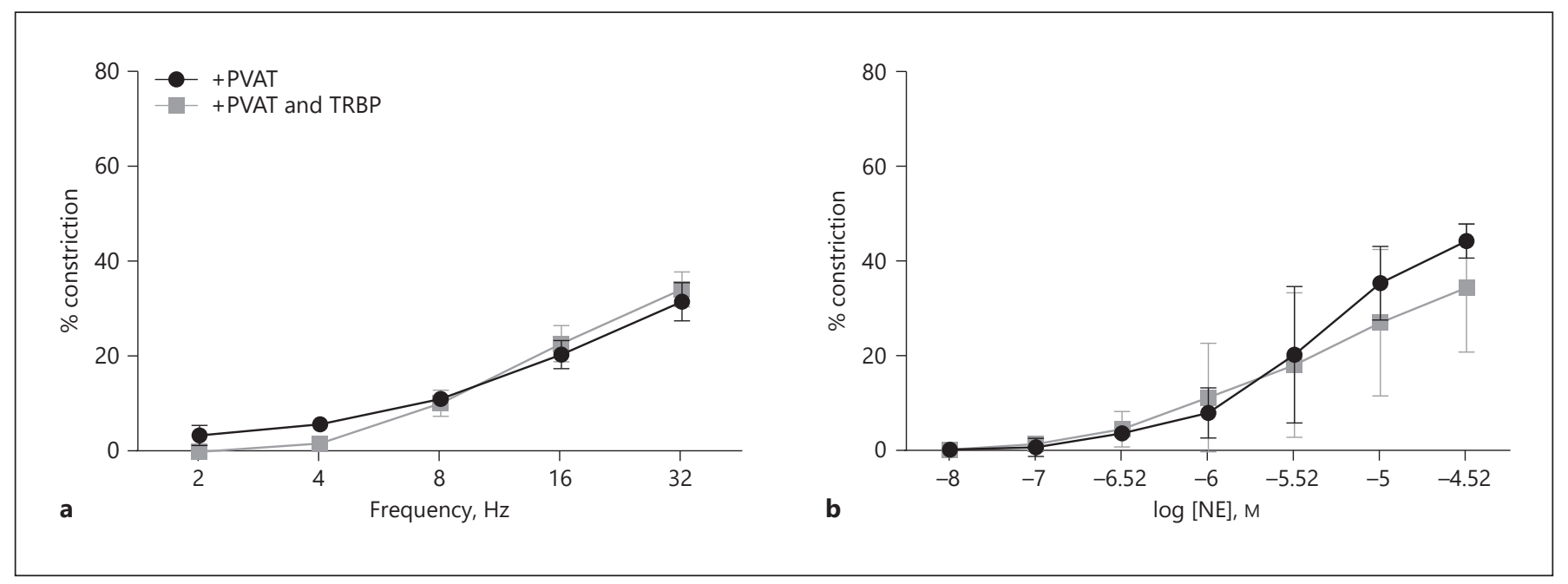

Fig. 8. TRBP had no effects on the PVAT anti-constrictive effect in vivo. Following control stimulations with EFS (a) and NE (b), PVAT-intact rat mesenteric arteries were incubated with TRBP $(3.5 \mu \mathrm{g} / \mathrm{mL})$ for $45 \mathrm{~min}$. Data shown are the mean \pm SEM. Differences in curves were tested using repeated-measures ANOVA $(n=3, p>0.05)$ with Bonferroni post hoc tests.

is used, the anti-contractile effect persists. Whilst this preparation was not used in the in vivo set up, it is unlikely that the PVAT is forming a barrier, as to properly visualize the artery walls in the in vivo set up the overlying PVAT must be removed, and the PVAT along the sides of the artery is not removed, but is gently pulled away to better visualize the vessel walls. This would suggest that both the anti- and proconstrictive effects of PVAT seen in this study are the result of vasoactive factors released by the PVAT into the surrounding solution.

The function of PVAT is generally considered to be anti-contractile; therefore, we expected a potentiation of vasodilation to Ach. Instead, we found that PVAT had a proconstrictive effect when challenged with Ach. This is consistent with previous findings in isolated mesenteric arteries [25]. Li et al. [25] attributed this effect to PVAT acting as a barrier and hindering diffusion of Ach; however, as discussed previously we do not believe that this is the case. Instead, these results might suggest that PVAT may also release a vasoconstrictor under appropriate conditions. There are a wide range of adipokines secreted by adipocytes [26]; therefore, it is quite possible that one of these may cause vasoconstriction, such as chemerin or visfatin, which have been shown to cause vasoconstriction in rat mesenteric arteries and aortae, respectively [27, 28]. Further experiments are required to identify the adipokine responsible, and whether the adipokine is acting as a procontractile factor, or is inhibiting mediators of the normal Ach response.

PVAT Effect in vivo

\section{Electrical Activation of Sympathetic Nerves in PVAT Exerts an Anti-Constrictive Effect via $\beta_{3}$-Adrenoceptors}

Recently, we have reported a functional role for $\beta_{3^{-}}$ adrenoceptors in the NE- and EFS-induced PVAT anticontractile effect in isolated mouse and rat mesenteric arteries in vitro $[12,21]$. Consistent with previous studies, the EFS-induced anti-constrictive effect in vivo was reduced by $\beta_{3}$-adrenoceptor inhibition, although the PVAT remained overall anti-constrictive. The remainder of the anti-constrictive effect is likely due to the NE transporter OCT3. As previously discussed, OCT3 transports NA into the adipocyte, thereby preventing NE from reaching the blood vessel and eliciting contraction [12]. To demonstrate this effect in vitro, the exogenous PVAT preparation described above was used, which allows us to remove the PVAT for pharmacological manipulation alone without interfering with NE transport in the blood vessel. It will be of interest to understand whether this mechanism is also relevant in vivo.

There was no effect of the $\beta_{3}$-adrenoceptor antagonist on the response to AVP, which reinforces previous suggestions that the anti-contractile mechanism may vary between different vasoconstrictors $[29,30]$. The enhanced anti-constrictive effect of PVAT in arteries stimulated with EFS incubated with the $\beta_{3}$-adrenoceptor agonist supports the hypothesis that adipocyte $\beta_{3}$-adrenoceptor activation is stimulating the release of a vasorelaxant. The lack of effect of the $\beta_{3}$-adrenoceptor agonist in NE-stim- 
ulated arteries is consistent with previous in vitro studies of rat mesenteric arteries [21], and suggests that the $\beta_{3^{-}}$ adrenoceptors are already saturated by the application of exogenous NE. With regards to AVP and Ach, whilst the ANOVA indicated a statistically significant difference in the overall means of the curves, the Bonferroni post hoc tests indicated no differences at individual points. This is likely due to the high degree of biological variation in these measurements, and warrants further investigation to definitively determine if $\beta_{3}$-adrenoceptor agonism affects the artery responses to AVP and Ach.

The lack of effect of the $\beta_{3}$-adrenoceptor antagonist on the response to AVP may suggest that there is little basal sympathetic tone in the PVAT. This is consistent with the limited tone of externalised arteries and transient drops in blood pressure [18], which may indicate low sympathetic outflow in this preparation.

\section{Adiponectin May Be the Anti-Constrictive Factor}

Released upon $\beta_{3}$-Adrenoceptor Activation in vivo

It has previously been reported that $\mathrm{ABP}$ reduces the NE- and EFS-induced PVAT anti-contractile effect in human and mouse arteries $[4,12,24]$, and measures of adiponectin secretion demonstrate that adiponectin is released from adipocytes upon activation of $\beta_{3}$-adrenoceptors [12]. Therefore, we tested the effects of ABP in our in vivo rat model. We found that the responses to EFS and NE were increased in PVAT-intact arteries, indicating that adiponectin may play a role in the nerve-evoked PVAT response. As a control for ABP, the effects of a blocking peptide for an unrelated protein were tested. We chose to examine the effects of TRBP, as both TRBP and ABP have a net charge of -1 , and are a similar length of amino acids. TRBP had no effect on PVAT function; therefore, we are confident that the effect of $A B P$ is specific. Circulating adiponectin is reduced in hypertension [13], and we have shown using tail cuff blood pressure measurements that the adiponectin knockout mouse is hypertensive [12]. These studies suggest that adipocytederived adiponectin may be essential in modulating vascular tone and blood pressure.

There are some studies which have reported an adiponectin paradox, whereby there exists a positive correlation between circulating adiponectin and advanced cardiovascular states such as stroke, coronary artery disease, and peripheral artery disease, as well as in chronic kidney disease and some cancers. However, recent reviews have criticised the experimental design of these studies, indicating that other confounding factors, such as age, BMI, and medications already being taken, were not considered [31, 32]. There are a vast number of studies indicating a cardioprotective effect of adiponectin in basic science studies using animal and cellular models, which makes the clinical correlation between adiponectin and mortality unexpected. It is possible that increased adiponectin secretion is the body's attempt at a protective response, and adiponectin resistance in multiple tissues such as adipose, skeletal muscle, liver, heart, and the vasculature results in the failure of this mechanism [33].

Clearly, the value of adiponectin as a clinical biomarker in cardiovascular disease is controversial and complex. Nonetheless, there is a clear reduction in circulating adiponectin in obesity and hypertension [14, 34-38]. In addition, anti-hypertensive treatments increase adiponectin expression in hypertensive patients [13]. It is these studies which bear the most relevance to the role of PVAT in blood pressure regulation, and to this study.

\section{Study Limitations}

The order of stimuli applied, i.e., EFS, NA, Ach, and then AVP, should be carefully considered. The same order was used in each experiment for this study, and it is possible that the order could influence results. However, long washout periods were used (30 $\mathrm{min})$, ensuring that the resting diameter of arteries in between each intervention was returned to baseline. In addition, as can be seen in Figures 4 and 6, where the $\beta_{3}$-adrenoceptor antagonist and agonist, respectively, were tested, the concentrationresponse curves were superimposable, despite a different history of the two curves. It therefore seems unlikely that the order of drugs has affected our results. Another limitation of the in vivo method is that it is not possible to run a time control artery side by side with the artery exposed to intervention. However, since the $\beta_{3}$-adrenoceptor agonist and antagonist had no effect in PVAT-denuded vessels, we are confident that the changes produced by these drugs in the presence of PVAT are genuine, and not a time effect.

\section{Conclusions}

PVAT was once thought to only provide mechanical support for the vasculature; however, it is becoming increasingly clear that PVAT is a highly, metabolically active, and complex tissue. This study has for the first time demonstrated a function for PVAT in modulating vascular tone in vivo, highlighting the physiological impor- 
tance of healthy PVAT. PVAT exerted an anti-constrictive effect upon electrical activation of sympathetic nerves, and upon stimulation with NE and AVP. In contrast, PVAT exerted a proconstrictive effect on the responses to Ach. Evidence suggests that $\beta_{3}$-adrenoceptors may contribute to sympathetic and noradrenergic PVAT function, and activation of these receptors stimulates the release of an anti-contractile factor, which may be adiponectin. Further dissection of the mechanisms of the PVAT effect in vivo is important for developing novel treatments of the vascular complications associated with PVAT dysfunction, namely obesity.

\section{Acknowledgments}

We would like to thank the animal unit at Aarhus University for their assistance with animal work.

\section{Statement of Ethics}

All experiments were approved by and conducted with permission from the Animal Experiments Inspectorate of the Danish Ministry of Justice.

\section{Disclosure Statement}

The authors have no conflicts of interest to declare.

\section{Funding Sources}

This work was funded in part by a grant from the British Heart Foundation FS/13/56/30645, the Marie Curie Initial Training Networks, SmArt and SmArtER, and Danish Council for Independent Research 7016-00013B.

\section{Author Contributions}

S.N.S., S.B.W., V.M., A.M.H., and C.A. were responsible for the conception and design of the research. S.N.S., S.B.W., J.N., and A.M. performed the experiments. S.N.S., S.B.W., V.M., A.M.H., and C.A. interpreted the results of the experiments. S.N.S. analysed the data, prepared the figures, and drafted the manuscript. S.N.S., S.B.W., A.M.H., and C.A. edited and revised the manuscript. All authors approved the final version of the report.

\section{References}

1 Aghamohammadzadeh R, Heagerty AM. Obesity-related hypertension: epidemiology, pathophysiology, treatments, and the contribution of perivascular adipose tissue. Ann Med. 2012 Jun;44(sup1 Suppl 1):S74-84.

2 Bjørndal B, Burri L, Staalesen V, Skorve J, Berge RK. Different adipose depots: their role in the development of metabolic syndrome and mitochondrial response to hypolipidemic agents. J Obes. 2011;2011:490650.

3 Szasz T, Webb RC. Perivascular adipose tissue: more than just structural support. Clin Sci (Lond). 2012 Jan;122(1):1-12.

4 Greenstein AS, Khavandi K, Withers SB, Sonoyama K, Clancy O, Jeziorska M, et al. Local inflammation and hypoxia abolish the protective anticontractile properties of perivascular fat in obese patients. Circulation. 2009 Mar; 119(12): 1661-70.

5 Gálvez-Prieto B, Dubrovska G, Cano MV, Delgado M, Aranguez I, González MC, et al. A reduction in the amount and anti-contractile effect of periadventitial mesenteric adipose tissue precedes hypertension development in spontaneously hypertensive rats. Hypertens Res. 2008 Jul;31(7):1415-23.
6 Bulloch JM, Daly CJ. Autonomic nerves and perivascular fat: interactive mechanisms. Pharmacol Ther. 2014 Jul;143(1):61-73.

7 Verma S, Lovren F, Pan Y, Yanagawa B, Deb $\mathrm{S}$, Karkhanis R, et al. Pedicled no-touch saphenous vein graft harvest limits vascular smooth muscle cell activation: the PATENT saphenous vein graft study. Eur J Cardiothorac Surg. 2014 Apr;45(4):717-25.

8 Kopjar T, Dashwood MR. Endoscopic versus "no-touch" saphenous vein harvesting for coronary artery bypass grafting: a trade-off between wound healing and graft patency. Angiology. 2016 Feb;67(2):121-32.

9 Aghamohammadzadeh R, Unwin RD, Greenstein AS, Heagerty AM. Effects of obesity on perivascular adipose tissue vasorelaxant function: nitric oxide, inflammation and elevated systemic blood pressure. J Vasc Res. 2015; 52(5):299-305.

10 Bussey CE, Withers SB, Aldous RG, Edwards G, Heagerty AM. Obesity-related perivascular adipose tissue damage is reversed by sustained weight loss in the rat. Arterioscler Thromb Vasc Biol. 2016 Jul;36(7):1377-85.
11 Withers SB, Forman R, Meza-Perez S, Sorobetea D, Sitnik K, Hopwood T, et al. Eosinophils are key regulators of perivascular adipose tissue and vascular functionality. Sci Rep. 2017 Mar;7(1):44571.

12 Saxton SN, Ryding KE, Aldous RG, Withers SB, Ohanian J, Heagerty AM. Role of Sympathetic Nerves and Adipocyte Catecholamine Uptake in the Vasorelaxant Function of Perivascular Adipose Tissue. Arterioscler Thromb Vasc Biol. 2018 Apr;38(4):880-91.

13 Yilmaz MI, Sonmez A, Caglar K, Celik T, Yenicesu M, Eyileten T, et al. Effect of antihypertensive agents on plasma adiponectin levels in hypertensive patients with metabolic syndrome. Nephrology (Carlton). 2007 Apr; 12(2):147-53.

14 Arita Y, Kihara S, Ouchi N, Takahashi M, Maeda K, Miyagawa J, et al. Paradoxical decrease of an adipose-specific protein, adiponectin, in obesity. Biochem Biophys Res Commun. 1999 Apr;257(1):79-83.

15 Gao YJ. Dual modulation of vascular function by perivascular adipose tissue and its potential correlation with adiposity/lipoatrophyrelated vascular dysfunction. Curr Pharm Des. 2007;13(21):2185-92. 
16 Gollasch M, Dubrovska G. Paracrine role for periadventitial adipose tissue in the regulation of arterial tone. Trends Pharmacol Sci. 2004 Dec;25(12):647-53.

17 Mikolajczyk TP, Nosalski R, Szczepaniak P, Budzyn K, Osmenda G, Skiba D, et al. Role of chemokine RANTES in the regulation of perivascular inflammation, T-cell accumulation, and vascular dysfunction in hypertension. FASEB J. 2016 May;30(5):1987-99.

18 Nyvad J, Mazur A, Postnov DD, Straarup MS, Soendergaard AM, Staehr C, et al. Intravital investigation of rat mesenteric small artery tone and blood flow. J Physiol. 2017 Aug; 595(15):5037-53.

19 Tandon S, Kambi N, Jain N. Overlapping representations of the neck and whiskers in the rat motor cortex revealed by mapping at different anaesthetic depths. Eur J Neurosci. 2008 Jan;27(1):228-37.

20 Søndergaard A, Overgaard C, Mazur A, Postnov D, Matchkov V, Aalkjaer C. Rat mesenteric small artery neurogenic dilation is predominantly mediated by $\beta 1$-adrenoceptors in vivo. J Physiol. 2019 Apr;597(7):1819-31.

21 Bussey CE, Withers SB, Saxton SN, Bodagh N, Aldous RG, Heagerty AM. $\beta 3$-Adrenoceptor stimulation of perivascular adipocytes leads to increased fat cell-derived $\mathrm{NO}$ and vascular relaxation in small arteries. Br J Pharmacol. 2018 Sep;175(18):3685-98.

22 Soltis EE, Cassis LA. Influence of perivascular adipose tissue on rat aortic smooth muscle responsiveness. Clin Exp Hypertens A. 1991; 13(2):277-96

23 Gao YJ, Zeng ZH, Teoh K, Sharma AM, Abouzahr L, Cybulsky I, et al. Perivascular adipose tissue modulates vascular function in the human internal thoracic artery. J Thorac Cardiovasc Surg. 2005 Oct;130(4):1130-6.
24 Lynch FM, Withers SB, Yao Z, Werner ME, Edwards G, Weston AH, et al. Perivascular adipose tissue-derived adiponectin activates $\mathrm{BK}(\mathrm{Ca})$ channels to induce anticontractile responses. Am J Physiol Heart Circ Physiol. 2013 Mar;304(6):H786-95.

25 Li R, Andersen I, Aleke J, Golubinskaya V, Gustafsson H, Nilsson H. Reduced anti-contractile effect of perivascular adipose tissue on mesenteric small arteries from spontaneously hypertensive rats: role of Kv7 channels. Eur J Pharmacol. 2013 Jan;698(1-3):310-5.

26 Edwards G, Félétou M, Weston AH. Endothelium-derived hyperpolarising factors and associated pathways: a synopsis. Pflugers Arch. 2010 May;459(6):863-79.

27 Watts SW, Dorrance AM, Penfold ME, Rourke JL, Sinal CJ, Seitz B, et al. Chemerin connects fat to arterial contraction. Arterioscler Thromb Vasc Biol. 2013 Jun;33(6): $1320-8$.

28 Yamawaki H, Hara N, Okada M, Hara Y. Visfatin causes endothelium-dependent relaxation in isolated blood vessels. Biochem Biophys Res Commun. 2009 Jun;383(4):503-8.

29 Fésüs G, Dubrovska G, Gorzelniak K, Kluge $\mathrm{R}$, Huang Y, Luft FC, et al. Adiponectin is a novel humoral vasodilator. Cardiovasc Res. 2007 Sep;75(4):719-27.

30 Withers SB, Bussey CE, Saxton SN, Melrose HM, Watkins AE, Heagerty AM. Mechanisms of adiponectin-associated perivascular function in vascular disease. Arterioscler Thromb Vasc Biol. 2014 Aug;34(8):1637-42.

31 Menzaghi C, Trischitta V. The adiponectin paradox for all-cause and cardiovascular mortality. Diabetes. 2018 Jan;67(1):12-22.
32 Woodward L, Akoumianakis I, Antoniades C. Unravelling the adiponectin paradox: novel roles of adiponectin in the regulation of cardiovascular disease. Br J Pharmacol. 2017 Nov; 174(22):4007-20.

33 Wang Y, Ma XL, Lau WB. Cardiovascular Adiponectin Resistance: The Critical Role of Adiponectin Receptor Modification. Trends Endocrinol Metab. 2017 Jul;28(7):519-30.

34 Hotta K, Funahashi T, Bodkin NL, Ortmeyer HK, Arita Y, Hansen BC, et al. Circulating concentrations of the adipocyte protein adiponectin are decreased in parallel with reduced insulin sensitivity during the progression to type 2 diabetes in rhesus monkeys. Diabetes. 2001 May;50(5):1126-33.

35 Tsioufis C, Kyvelou S, Dimitriadis K, Syrseloudis D, Sideris S, Skiadas I, et al. The diverse associations of uric acid with lowgrade inflammation, adiponectin and arterial stiffness in never-treated hypertensives. J Hum Hypertens. 2011 Sep;25(9):554-9.

36 De Rosa A, Monaco ML, Capasso M, Forestieri P, Pilone V, Nardelli C, et al. Adiponectin oligomers as potential indicators of adipose tissue improvement in obese subjects. Eur J Endocrinol. 2013 Jun;169(1):37-43.

37 Meijer RI, Bakker W, Alta CL, Sipkema P, Yudkin JS, Viollet B, et al. Perivascular adipose tissue control of insulin-induced vasoreactivity in muscle is impaired in $\mathrm{db} / \mathrm{db}$ mice. Diabetes. 2013 Feb;62(2):590-8.

38 Hung J, McQuillan BM, Thompson PL, Beilby JP. Circulating adiponectin levels associate with inflammatory markers, insulin resistance and metabolic syndrome independent of obesity. Int J Obes. 2008 May;32(5):772-9. 